\title{
Do Under-Managed Portfolios Outperform Over-Managed Portfolios?
}

Hamid Z. Ahmadi, (E-mail: ahmadih@csus.edu), California State University, Sacramento

Janna Khoroujik, California State University, Sacramento

Rafiqul B. Rafiq, California State University, Sacramento

\begin{abstract}
How often should a portfolio be rebalanced? This is the question our study attempts to answer. The Internet stock bubble and its aftermath made portfolio mangers very sensitive to their management styles. Specifically, they had to reassess how often to evaluate a client's portfolio. This work examines the performance of portfolios that were aggressively managed and compares their riskadjusted returns with those of portfolios that were managed infrequently. To accomplish this, we change the rebalancing frequency of a well-diversified portfolio and track its performance over time. This study will not only enable us to determine whether the performance of an actively-managed portfolio surpasses the performance of an under-managed or unmanaged portfolio, but it will also allow us to determine the optimal rebalancing period for maximizing risk-adjusted returns. The asset selection and portfolio optimization methodologies applied to the portfolios in this study are identical to maintain consistency and comparability of results. To evaluate the performance of each portfolio, we used daily observations from September 2000 to September 2006 and applied various rebalancing frequencies using the QuantAnalysis application at www.fundsformation.com.
\end{abstract}

\section{INTRODUCTION}

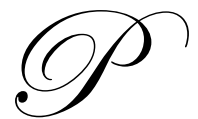

ortfolio managers interpret the term "rebalancing" in two different ways. To some managers, rebalancing means the reallocation of funds among different asset classes. For instance, if 80 percent of a portfolio's assets are invested in equities and 20 percent are invested in bonds, the manger can rebalance the portfolio in response to changing market conditions and reduce the percentage invested in equities to 70 percent. To other managers, rebalancing means keeping the percentages invested in each asset at an optimal level and rebalancing the portfolio if the percentages deviate from that optimal allocation. This ensures that the portfolio's risk exposure is not far different from what the manager originally intended. In this case, managers are more concerned about how often they should review their portfolios and rebalance the percentages. In our work, we are considering "rebalancing" in the latter sense and seeking to determine if there is an optimal rebalancing period that managers can use to ensure their clients' portfolios remain optimized.

The terms "over managed" and "under-managed" may also be interpreted differently by different managers and thus warrant clarification. Customarily, portfolios that are evaluated at least once a year are considered "managed portfolios." This one year evaluation frequency is rooted in tax and other annual reporting requirements. Thus, for purposes of this study, any portfolio that is not evaluated at least once a year is considered an "under-managed" portfolio. Conversely, a portfolio that is evaluated weekly or even daily is considered aggressively-managed or "overmanaged." In this study, we examined the performance of portfolios with different rebalancing frequencies (e.g. biweekly, monthly and bimonthly) to determine the optimal rebalancing period for a well-diversified portfolio.

After an investor decides how to allocate his or her assets, the decision as to how often to rebalance a portfolio is probably the most important factor in creating and maintaining a successful investment. So what type of rebalancing approach should a portfolio manager adopt? For example, should he take a laissez-faire approach and only rebalance every 18 months or should he aggressively manage the portfolio and rebalance on a daily basis? The answer to this question is partially subjective and partially determined by factors such as the type of assets in the portfolio, the investment strategy and the amount of work required to rebalance the portfolio. 


\section{REBALANCING APPROACHES AND FACTORS}

In general, there are two approaches to portfolio rebalancing: calendar rebalancing and threshold rebalancing. With calendar rebalancing, which is the most common and most disciplined rebalancing method, an investor chooses a particular frequency or time period, such as quarterly, semiannually or annually, to rebalance. On the other hand, threshold rebalancing requires that a portfolio be rebalanced when an asset deviates from its optimal percentage by a specified number. Rebalancing is only triggered once this "threshold" has been crossed. For example, a five percent rebalancing threshold would require that a portfolio be rebalanced whenever one asset is five percent above or five percent below a specified target. The most common approach to measuring the effects of rebalancing is to compare a periodically-balanced portfolio, such as mandatory monthly rebalancing, against a never-rebalanced portfolio and then chart the differences in returns.

Having decided on the rebalancing approach, one needs to recognize that there are several important factors that may have an effect on the frequency of portfolio rebalancing. The most significant factors include asset type, the investor's risk tolerance, catastrophic events, transaction costs and taxes.

One of the most important factors in the rebalancing decision is what is included in one's portfolio. Aggressive investments in technology stocks require constant attention from the portfolio manager as the stocks of such companies can fluctuate significantly. On the other hand, "safe" stocks, such as utility companies, produce steady returns over time and are generally less vulnerable to market fluctuations need less management. Therefore, the type of stock and the industry have an enormous influence on rebalancing decisions.

The type of assets in a portfolio reflects the personality of the investor who owns them. In fact, the stocks an investor holds in his portfolio often reveal his risk tolerance, which is another influence on the rebalancing frequency. Should the investor be more conservative, he is likely to pick "stable" stocks and does not need to monitor his investment very closely.

Although they rarely occur, catastrophic events can cause an investor to rotate assets from one class to another, thus changing the rebalancing frequency. However, such events are rare and would be considered anomalies if we were to include them in this study. Thus, they are excluded from our analysis.

Additionally, transaction costs and taxes can deter investors from rebalancing, despite the fact that rebalancing may be sorely needed. This occurs when an investor reasons that frequent rebalancing might eliminate any profit he stands to gain from the portfolio unless the amount is significant enough to offset the transaction costs. Also, since the long-term capital gains tax rate is lower than the rate for short-term gains, investors often prefer to postpone rebalancing rather than to pay more taxes. However, studies show that taxes do actually play a more complex role in dissuading the investor from frequent rebalancing. While there is no consensus on this particular matter, the most important factor in determining rebalancing frequency appears to be the components of the portfolio.

\section{LITERATURE REVIEW}

Although the need for portfolio rebalancing is generally acknowledged, very little research has been published on this topic and on the optimal rebalancing frequency, in particular. However, several studies are instructive as we seek to identify this optimal frequency. Of perhaps most relevance to our study, Arnott and Lovell (1993) found that, for the years 1968 to 1991, their portfolio produced a return of 9.02 percent with annual rebalancing, 9.12 percent with quarterly rebalancing and 9.16 percent with monthly rebalancing. Thus, they concluded that monthly rebalancing was preferable to less active rebalancing approaches. At the same time, they found that calendar rebalancing produced somewhat greater gains than threshold rebalancing.

In support of less frequent rebalancing, Eaker and Grant (2002) analyzed the effects of equity portfolio rebalancing in nine emerging markets. They concluded that the benefits of rebalancing decrease with the higher rebalancing frequency and found that semi-annual rebalancing approach outperformed the monthly rebalancing by 2.62 percent per year. 
Addressing one of the factors that influence rebalancing, Parker Hall III (2005) suggested that although catastrophic events influence portfolio's performance, effective rebalancing approach helps the portfolio to recover sooner.

Donohue and Yip (2003) compared several rebalancing approaches and incorporated the transaction costs. Among calendar, threshold and no rebalancing, they found that the absence of rebalancing outperforms other strategies with significantly higher risk. In addition, they discovered that annual rebalancing incurred the same transaction costs as threshold rebalancing while generated lower returns.

Finally, several studies focused on investors' motivations for rebalancing and the timing of such activity. For example, Chan (1985), DeBondt and Thaler (1987) found that investors rebalance their portfolios in order to realize losses for tax purposes. Ritter (1988) studied the effects of seasonal portfolio rebalancing by individuals and presented evidence that the buy-sell ratio for individual investors is below normal in December and above normal in January.

\section{DATA AND CRITERIA}

In order to avoid problems related to the portfolio's components that may affect rebalancing frequency, we have selected the 30 stocks in the Dow Jones Industrial Average, which are generally stable and represent different industries. Also, such a selection represents a diversified portfolio with average market risk. The rebalancing options chosen range from very active (weekly and biweekly) to less active (bimonthly and quarterly). For the results to be statistically significant and to ensure we have at least 30 observations for the quarterly rebalancing terms, we collected daily observations from September 2000 to September 2006. We used the DataGrabber application at www.fundsformation.com to achieve this task.

Table 1: Dow Jones Industrial Average Components

\begin{tabular}{|c|c|c|c|c|c|}
\hline Ticker & Name & Industry & Ticker & Name & Industry \\
\hline AA & Alcoa Inc. & Aluminum & JNJ & Johnson \& Johnson & Drug Manufacturers - Major \\
\hline AIG & AIG-AMERIC.INT.GRP & Property \& Casualty Insurance & JPM & JPMorgan Chase & Money Center Banks \\
\hline AXP & American Express Company & Credit Services & KO & The Coca-Cola & Beverages - Soft Drinks \\
\hline BA & Boeing & Aerospace/Defense & MCD & McDonald's & Restaurants \\
\hline C & Citigroup & Money Center Banks & MMM & 3M Company & Conglomerates \\
\hline CAT & Caterpillar & Farm \& Construction Machines & MO & ALTRIA GROUP & Cigarettes \\
\hline DD & DU PONT DE NEMOURS & Chemicals - Major Diversified & MRK & MERCK AND CO. & Drug Manufacturers - Major \\
\hline DIS & The Walt Disney & Entertainment - Diversified & MSFT & Microsoft & Application Software \\
\hline GE & GENERAL ELECTRIC & Conglomerates & PFE & Pfizer Inc. & Drug Manufacturers - Major \\
\hline GM & GENERAL MOTORS & Auto Manufacturers - Major & PG & PROCTER GAMBLE & Cleaning Products \\
\hline HD & The Home Depot & Home Improvement Stores & T & AT\&T Inc. & Telecom Services - Domestic \\
\hline HON & Honeywell International Inc. & Conglomerates & UTX & UNITED TECHNOL & Conglomerates \\
\hline HPQ & Hewlett-Packard Company & Diversified Computer Systems & VZ & Verizon & Telecom Services - Domestic \\
\hline IBM & IBM & Diversified Computer Systems & WMT & Wal-Mart Stores, Inc. & Discount, Variety Stores \\
\hline INTC & Intel Corporation & Semiconductor - Broad Line & XOM & Exxon Mobil & Major Integrated Oil \& Gas \\
\hline
\end{tabular}

\section{THE MODEL}

There are three common approaches to portfolio optimization - the Equal Weights (EW) approach, the Minimum Variance Portfolio (MVP) approach, and the Modern Portfolio Theory (MPT) approach. While each of these methods can help a portfolio manager respond to his clients' needs, there is a powerful model that can be used to generate an optimal portfolio - the Risk-Adjusted Managed Portfolio (RAMP) model. RAMP is the model we used in this study. While the MVP and MPT approaches rely on variance to determine risk and help portfolio managers determine whether to buy or sell a particular asset, RAMP considers beta and variance. Specifically, RAMP is a twostage model that provides us with a simple, but effective means of selecting stocks and determining the appropriate proportions of each to hold in our portfolio. The first stage of the RAMP model uses the Treynor Index to help us 
select stocks for our portfolio, while the second stage uses the Sharpe Index to determine what proportion of our portfolio should be invested in each stock.

\section{Stage 1 -Stock Selection}

Since we need to determine which stocks to include in our optimal portfolio, the first stage of the RAMP model assists us with stock selection. Of course, it would be very easy to form such a portfolio if there were a single number we could use to decide whether or not to include a particular stock in the portfolio. The RAMP optimization model finds this key number and tells us to include in our portfolio any stock with a Treynor Index that exceeds it.

TreynorIndex $=\frac{\left(R_{j}-R_{f}\right)}{\beta_{j}}$

Where $R_{j}$ is the rate of return for stock " $\mathrm{j}$," $R_{f}$ is the risk-free rate of return and $B_{j}$ is the beta value for stock “j”. Note that both the market rate of return $R_{m}$ and the risk-free rate of return $R_{f}$ result from general stock market fluctuations and are not due to the work of a portfolio manager. In other words, $\left(R_{m}-R_{f}\right)$ is the return of an "unmanaged portfolio." If the Treynor Index $\frac{\left(R_{j}-R_{f}\right)}{\beta_{j}}$, which is the risk-adjusted return of stock $j$, is greater than $\left(\boldsymbol{R}_{m}-\boldsymbol{R}_{f}\right)$, then stock $\boldsymbol{j}$ outperformed the unmanaged market portfolio and stock $\boldsymbol{j}$ is a candidate to be included in our optimal portfolio. Once again, the general rule for including or excluding stocks from our optimal portfolio is to include the stock if its Treynor Index is greater than the key number calculated by RAMP. We will call this key number the cut-off number.

\section{Stage 2 - Determining Optimal Proportions}

Inclusion and exclusion of stocks is the first part of the RAMP model. The second task is to find the proportions to be invested in each included stock in order to create an optimal portfolio. This optimization process is similar to MPT in that the proportions of the market portfolio, or Portfolio M, are determined by maximizing the slope of the Capital Market Line (CML). In other words, we hold stocks in the proportions dictated by the maximum Sharpe Index.

In summary, the RAMP model compares a stock's risk-adjusted return (as measured by the Treynor Index) with the cut-off number mentioned above to determine whether to include the stock in our portfolio. It then uses the Sharpe Index to find the proportion of funds to be invested in each stock in order to create an optimal portfolio. Thus, the optimal portfolio we select using the RAMP model will not only have passed the Treynor Index test, but will also have secured the highest possible return at any given level of risk.

\section{Parameters}

Our data consists of daily observations from September 2000 to September 2006. We split the data into two parts: 1) observations from September 2000 to August 2005; and 2) observations from September 2005 to September 2006. The first part is used to compute inputs for the RAMP optimization model and the second part is used to track the performance of the portfolios.

As mentioned above, the RAMP model includes a stock if its Treynor Index is greater than a cut-off number. One of the inputs to the Treynor Index is the risk-free rate and in this study we use an aggregate rate of 3 percent for this parameter. In addition, we selected the option to pay a two percent money market rate to the unused cash in the account. 


\section{RESULTS}

As mentioned above, Arnott and Lovell examined annual, quarterly and monthly rebalancing periods and concluded that quarterly rebalancing produced a higher return than either annual or monthly rebalancing. Our results, which are based on an anlaysis of weekly and biweekly rebalancing in addition to the frequencies studied by Arnott and Lovell, show that from September 2005 to September 2006 quarterly rebalancing produced a higher return than more frequent rebalancing (please see Graph 1 and Table 2 below). On average, the return of the quarterly rebalanced portfolio was 6.32 percent higher than that of the weekly rebalanced portfolio and 3.62 percent higher than that of the monthly rebalanced portfolio. As Graph 1 illustrates, our study also found that quarterly rebalancing produced a much higher return over this time period than the S\&P 500, which we used as our stock market performance benchmark.

\section{Graph 1}

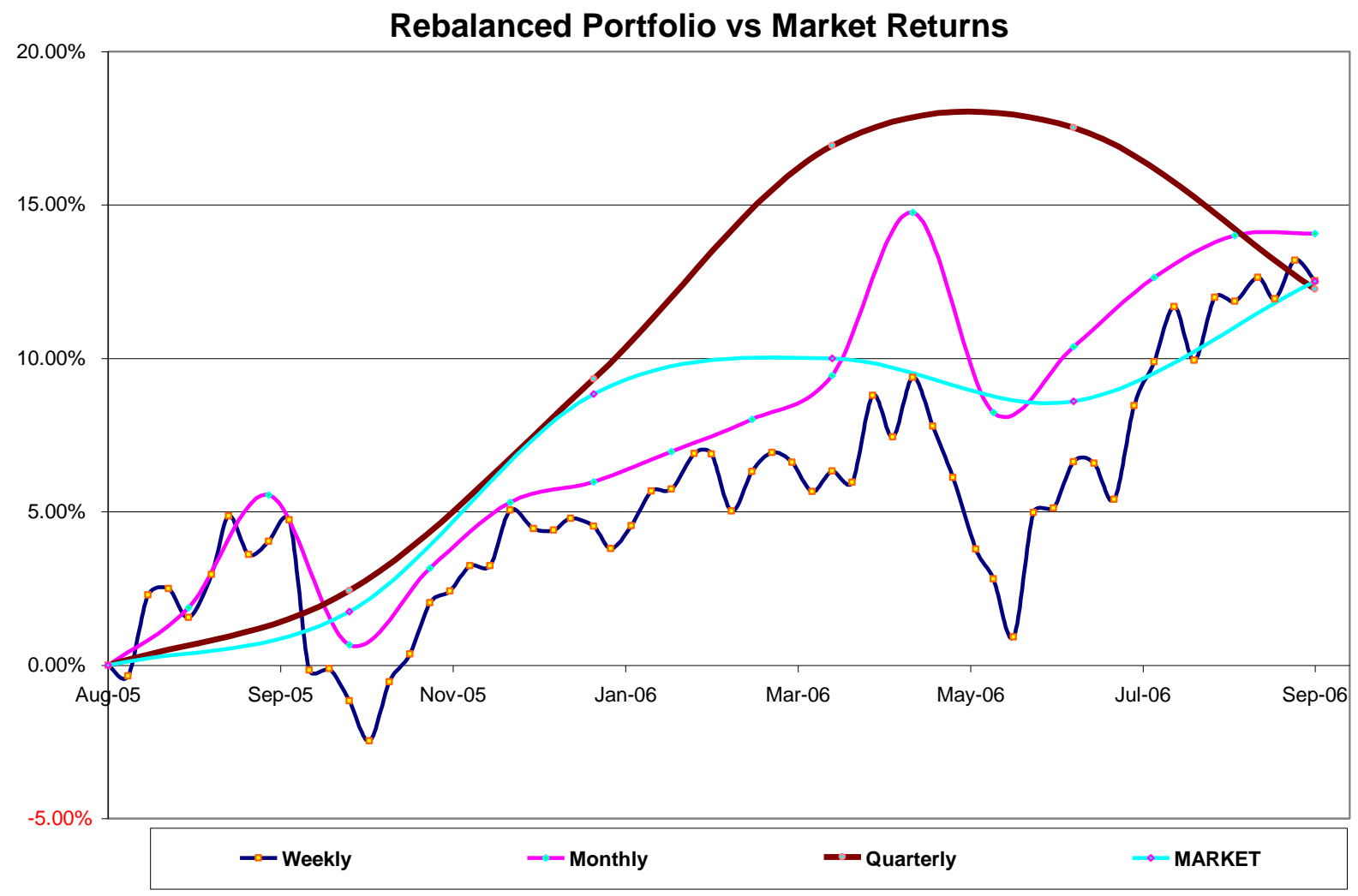

Table 2: Rebalancing Summary

\begin{tabular}{|c|c|c|c|}
\hline & Weekly & Monthly & Quarterly \\
\hline Average Return & $5.37 \%$ & $8.07 \%$ & $11.69 \%$ \\
\hline Standard Deviation & $3.69 \%$ & $4.48 \%$ & $6.18 \%$ \\
\hline Relative Risk (CV) & 0.69 & 0.56 & 0.53 \\
\hline
\end{tabular}

Graph 2 shows the performance of the portfolio with weekly, biweekly, monthly, bimonthly, and quarterly rebalancing frequencies. This graph clearly shows that, as the frequency of rebalancing decreases, the return of the portfolio increases. That is, weekly rebalancing produces high volatility and results in the lowest average return. However, as we rebalance less frequently, the portfolio's volatility decreases and performance improves. 


\section{Graph 2}

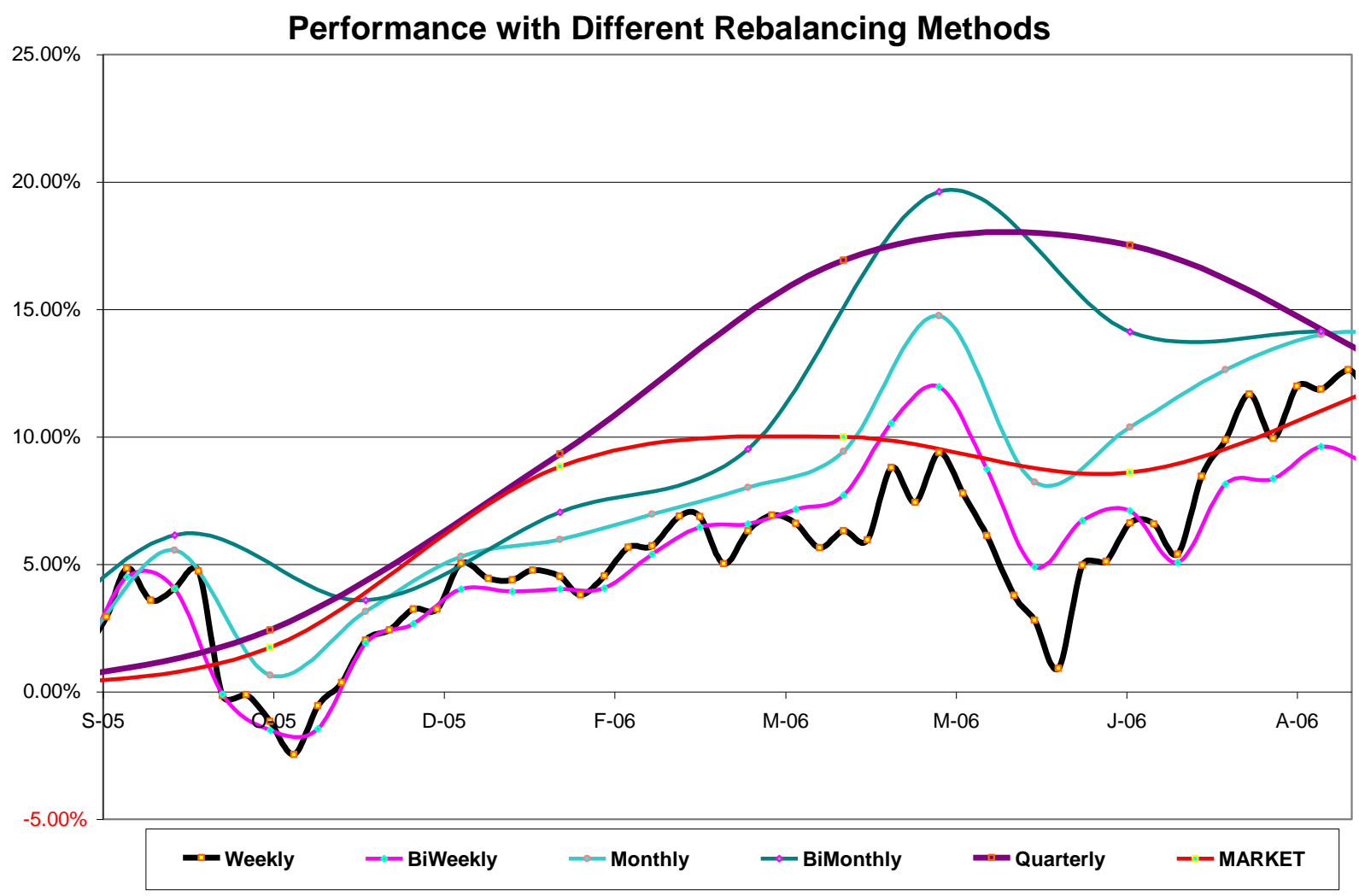

Table 3: Rebalancing Summary

\begin{tabular}{|c|c|c|c|c|c|}
\hline & Weekly & BiWeekly & Monthly & BiMonthly & Quarterly \\
\hline Average Return & $5.37 \%$ & $5.39 \%$ & $8.07 \%$ & $10.60 \%$ & $11.69 \%$ \\
\hline Standard Deviation & $3.69 \%$ & $3.39 \%$ & $4.48 \%$ & $5.61 \%$ & $6.18 \%$ \\
\hline Relative Risk (CV) & 0.69 & 0.63 & 0.56 & 0.53 & 0.53 \\
\hline
\end{tabular}

\section{CONCLUSIONS AND QUALIFICATIONS}

This work applied different rebalancing frequencies to a well-diversified portfolio and compared the riskadjusted returns achieved with each of these frequencies. The results indicate that a quarterly-rebalanced portfolio will outperform a portfolio that is rebalanced more frequently. Our study also shows the increased volatility of returns associated with over-managed portfolios. Considering the outcome of this work and that of Arnott and Lovell's, which stated that quarterly rebalancing produces higher returns than either annual or monthly rebalancing, we conclude that the optimal rebalancing frequency is quarterly.

Additionally, to further advance research in this subject matter, future studies should consider the effect of transaction costs and taxes on rebalancing decisions. We also believe more studies on rebalancing need to consider portfolios with different asset classes to make the choices of the portfolio manager more precise. 


\section{REFERENCES}

1. Ahmadi, H. \& Galindo, C. (2005). Two Staged Portfolio Optimizations. Journal of Business \& Economics Research, Vol. 3, No. 4.

2. Ahmadi, H. \& Soriano, B. C. (2003). An Analytical Technique for Determining Efficient Portfolios. American Academy of Accounting and Finance, Proceedings.

3. Ahmadi, H. (1996). Testability of Arbitrage Pricing Theory by Neural Networks. Neural Networks in Finance and Investments. Probus Publishing Company, Chicago.

4. Arnott, R. \& Lovell, R. (1993, Spring). Rebalancing: Why? When? How Often? The Journal of Investing, Vol. 2, No. 1: 5-10.

5. Chan, C. (1985, December). Can Tax Loss Selling Explain the January Seasonal in Stock Returns? Journal of Finance, Vol. 41, 1115-28.

6. Debondt, W. \& Thaler, R. (1987, July). Further Evidence on Investor overreaction and Stock Market Seasonality. Journal of Finance, Vol. 42, 557-81.

7. Donohue, C. \& Yip, K. (2003, Summer). Optimal Portfolio Rebalancing with Transaction Costs. Journal of Portfolio Management, Vol. 29, No. 4: 49.

8. Eaker. M. R. \& Grant, D. (2002). The Wealth Effects of Portfolio Rebalancing in Emerging Equity Markets. Journal of Multinational Financial Management, Vol. 12, 79-88.

9. Khoroujik, J. \& Ahmadi, H. (2006). The Empirical Evidence Conclusively Supports Global Investing. International Business \& Economics Research Journal. Forthcoming.

10. Parker Hall III, J. (2005, May-June). Good News! Financial Analysts Journal, Vol. 61, No.3: 42.

11. Ritter, J. (1988, July). The Buying and Selling Behavior of Individual Investors at the Turn of the Year. Journal of Finance, Vol. 43, 701-17. 
NOTES 